\title{
Information Support of Efficiency of the Transportation Process
}

\author{
Vladimir Baskov ${ }^{1}$, and Ekaterina Isaeva $^{1^{*}}$ \\ ${ }^{1}$ Yuri Gagarin State Technical University of Saratov, 77 Politekhnicheskaya str., Saratov, 410054, \\ Russia
}

\begin{abstract}
The use of information technology affects the congestion of the transport network. Coordinated control is designed to ensure non-stop passage of a group of cars through all the regulated intersections of the highway at a certain speed. As part of the application of the latest information technologies, the following indicators of the quality of transportation can be improved: timeliness of transportation, transportation safety, economic indicators, transportation speed, information services.
\end{abstract}

\section{Introduction}

The development of the transport infrastructure leads to an increase in the quality of transportation and road safety based on modern information technologies. This is due to the versatility of road transport, which is primarily aimed at consumers, both public and individual. More than half of the volume of cargo and passengers is transported by road.

However, taking into account the growth of motorization and the improvement of the quality of transport services, a number of significant problems appear: an increase in the number of road accidents (RTA), the formation of traffic jams during peak hours, loss of time for road users, high consumption of fuels and lubricants (POL), increased harm to the environment and, ultimately, increased operating costs for road transport.

\section{Material and methods}

The disorganized traffic flow on the street and road network of cities is one of the reasons for the formation of congestion. For the effective functioning of the traffic mechanism in many large cities, intelligent transport systems (ITS) are used, which provide information support for the transportation process (Smart City).

Many cities and towns around the world are turning to smart social media devices to solve urban problems [1], such as traffic congestion, pollution, health care, safety monitoring, to improve living standards for the daily comfort of the population. Intelligent sensors that are installed throughout the city, in vehicles, in buildings, on the roads, in control systems, security monitoring, as well as in applications and devices that are used by people living or working in the city [2]. Providing information to the public that harnesses

* Corresponding author: katherina3@mail.ru 
these high-tech capabilities of smart cities. Big data analytics is used to make decisions about the planning of public spaces, how to make the best use of their assets, and how to communicate administrative notices in a more efficient, viable and appropriate manner [3].

Thus, most cities have invested heavily in information and communication technology (ICT) infrastructure over the past decades, including computers, broadband accessibility, and some sensor systems [4].

In addition, there was a surge in the proliferation of sensors, along with the development of adaptive systems, there were modern advances in the field of sensor systems in the Internet of Things [5]. The benefits of social communication and the spread of the Internet of Things (IoT) for smart urban areas are now widely used [6].

In general, implementing smart cities is a broad concept, which is why this article focuses on the transport sector.

Current analysis of smart city data implies complex streaming analytics for a complete set of actions aimed at achieving real results [7]. The analysis includes the following contributions:

1) Analysis of thousands of traffic congestion cases, road capacity measures, traffic signalling and dynamic, consistent information to better inform citizens.

2) Events, episodic road surveys using real data collected by citizens, devices and sensors.

3) Turning web pages into information from social networks, analysis of important city events, assumptions, research and much more. Consolidation of information from physical (sensors / devices) and social sources (social organizations) can provide complete, important information and add to more accurate estimates and knowledge.

Smart cities will emerge as new areas of focus for transportation, utilities, smart buildings and smart security (Figure 1).

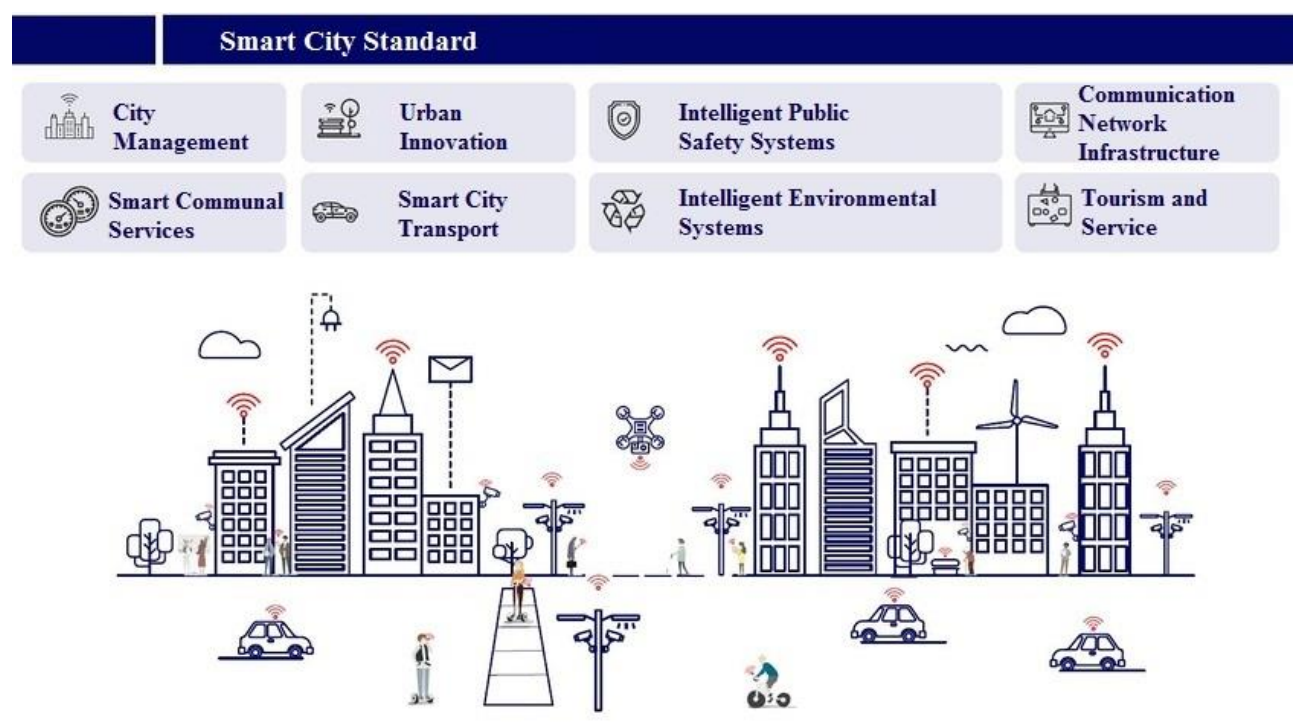

Fig. 1. Smart City Standard.

The most correct way to implement a smart city is to create an intelligent system.

Key smart city resources: transportation, climate change, energy, utilities, security surveillance, healthcare, business management, etc.

Likewise, reliable city traffic analysis, security investigations and information on traffic blocking and roadside sensors can be used to change time delays, misinterpretations of security observations, speed limit points and city fare tax, monitor safety monitoring and 
traffic flow in greater numbers. streams for long term results. To guide traffic around incidents, sensors additionally record street conditions, weather updates, building structures, traffic lights and annexes to support maintenance planning when needed.

Developing a roadmap for smart cities is based on four pillars: Connectivity is the foundational layer of a smart city. Real-time data is collected about people, places and things using smart sensors, and this data is stored on cloud application servers for analysis and use for more efficient decisions and planning in real time.

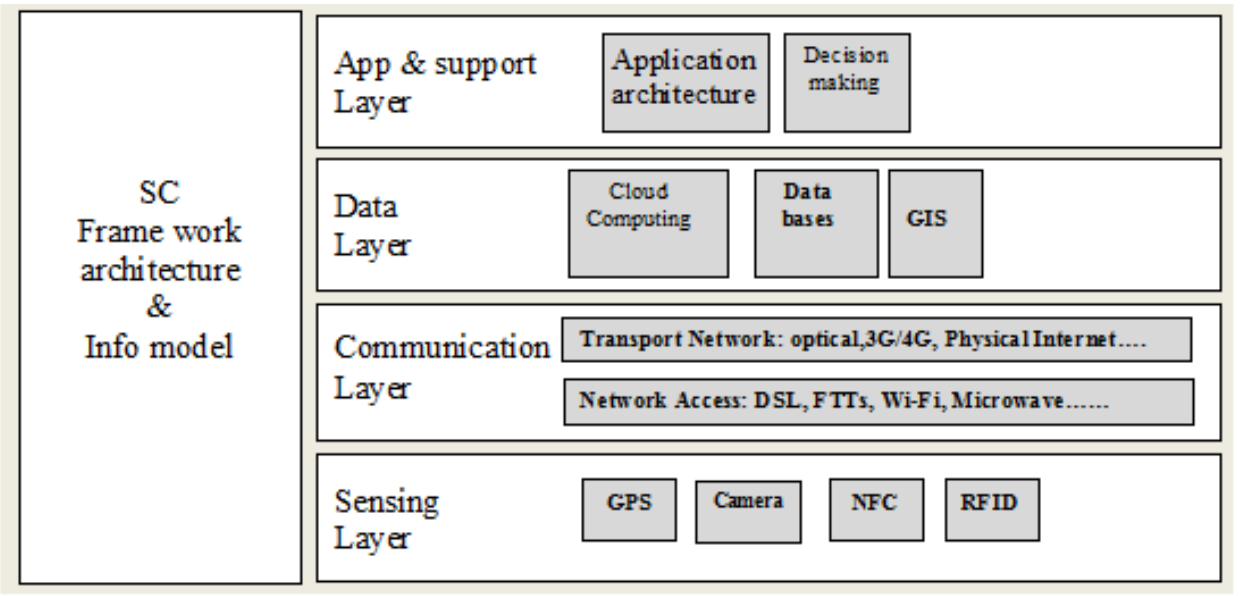

Fig. 2. Multi-layered model of a smart city.

The adaptive data analysis phase is displayed in the background. It consists of different levels, lower level (device, communication plane), middle levels (data, information validation) at higher levels (application, dashboard plane). At each level, individual pieces of code perform specific operations related to data collection, messaging, data access, semantic annotation, exploration, or perception, where applications can attach to segments from different levels in light of their specific preconditions. Along with these advances, they can be connected and work mainly in smart city applications. The current breakthrough advances in handheld smart phones, interpersonal organizational services and facilities are coordinated together for a new time machine and machine and human communication [8].

The main components of the model The multi-level wireframe model of a smart city, shown in Fig. 2, has four main levels, described below as:

a) Sensitivity level The sensor level comprises tens to thousands of sensor hubs connected using intelligent remote technologies. They collect data from the environment and transfer it to other connected devices, which transfer the data to a cloud server over the Internet.

b) Communication layer

The innovative Wireless Heart technology provides superior remote protocol access to the full range of processor, control and resource management capabilities for applications. DigiMesh is an exclusive shared systems network topology for use when connecting to a remote network endpoint over the physical Internet.

c) Data layer

Data capacity and processing should be possible at the edge of the network itself or on a cloud server. If any pre-processing of the data is needed, it is usually done either on the sensor or on another nearby device.

The processed data is then regularly sent to a remote server. The capacity and processing capability of an IoT object is further controlled by the available assets, which 
are typically used exclusively in exceptional cases due to capacity, survivability, control, and computational constraints.

d) Application layer

The application layer is responsible for organizing and presenting data. The application layer on the Internet is regularly governed by the Hypertext Transfer Protocol (HTTP) / File Transfer Protocol (FTP) standards. The proposed developments in this study are dynamic data exchange with clients using mobile phones as a specific device. It may be that HTTP is not practical in resource provisioning situations because it is relatively verbose and introduces significant parsing overhead.

In our country only certain elements of the ITS are used, but even in this case, there is a tendency of efficient use of the transport network and the introduction of such systems in the transportation process. Analyzing the experience of foreign countries in the application of ITS, use for the transportation process and traffic management, we can draw a conclusion that they are effective and expedient for use in road transport.

ITS increases the efficiency of vehicle use, reduces fuel consumption, reduces harmful emissions into the atmosphere, by organizing traffic flows with reduction of transport delays.

In conditions of overloaded transport networks of cities and in terms of improving the efficiency of traffic management, the Moscow City Government approved the ITS Development Concept (figure 3).

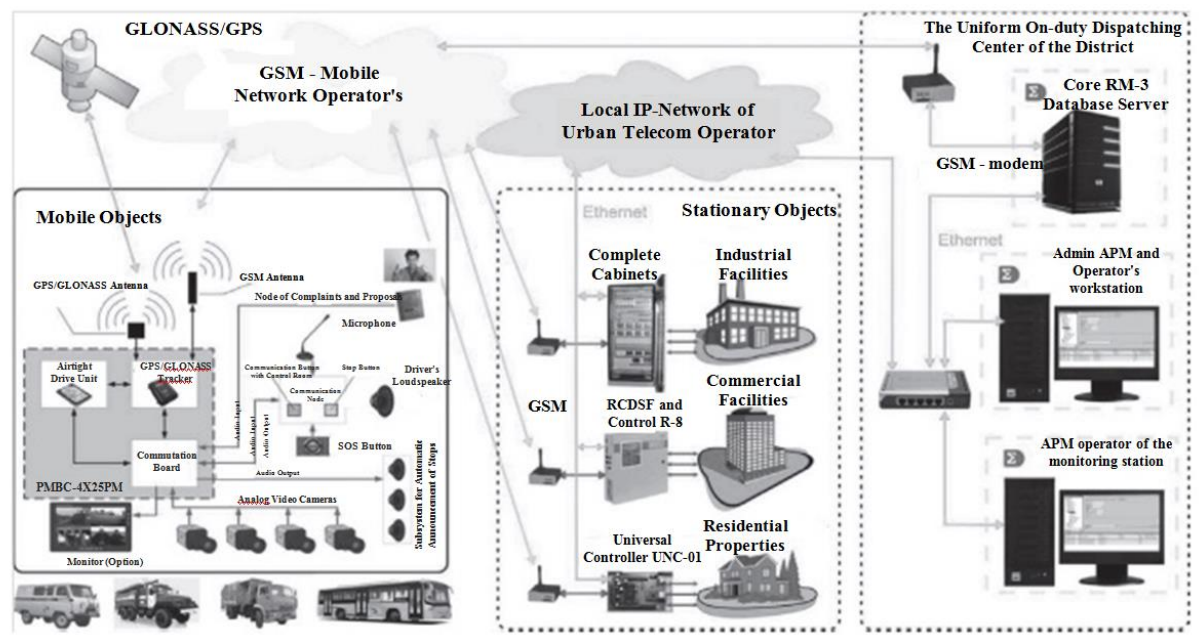

Fig. 3. General ITS structure of the city.

For optimal implementation of the Concept provisions, it is necessary to create a single intellectual system so that it can: collect data on traffic flow parameters; automatic detection of incidents; automatic detection of traffic violations; collection of data on the availability of free parking spaces; control over the movement of the fixed route transport; control over the movement of motor vehicles; transfer of statistical data to mobile electronic devices; functioning of the geoinformation resource.

Despite the great potential of ITS, software products are still not all present in the market. Automobiles are also rarely equipped with telematics systems. It is assumed that the introduction of modern software and the transmission of data on traffic parameters in real time can reduce traffic jams in urban areas by $40 \%$, which will positively affect the efficiency of vehicle use and the quality of transportation (Figure 4).

In solving problems of traffic management and the process of transportation, specialists face the main problem: measuring the importance of measures that contribute to improving 
the functioning of transport and pedestrian flows. It is necessary to compare the importance of these activities and give them an integrated assessment. For accurate estimation, the data obtained in the course of full-scale and computational experiments are used [9,10]. A fullscale experiment allows you to collect the necessary information by measuring in real-time conditions.

Mathematical modeling, applicable to the computational experiment, is usually divided into analytical and simulation. Taking into account the complexity of the processes of managing the transportation of passengers and cargo, in analytical modeling, in order to simplify, they resort to severe restrictions. In this connection, the analytical model makes it possible to find an approximate solution of the problem. This leads to a lack of constructive research results that make it impossible to use them in the design of ITS. The simulation model, unlike the analytical one, makes it possible to control traffic flow of varying complexity with the required level of detail [11].

A transport flow is a complex control object that requires the creation of a control object model, i.e. model of traffic flow [12]. The transport flow, as a model, includes a large number of discrete elements (i.e. vehicles). Specialists in this field on the basis of their traffic research $[13,14,15]$ have developed a set of measures and criteria [16]. The most commonly used indicators for road traffic assessment are traffic intensity $(\mathrm{v} / \mathrm{h})$, speed $(\mathrm{km} /$ h) and density of transport flow (v/m).

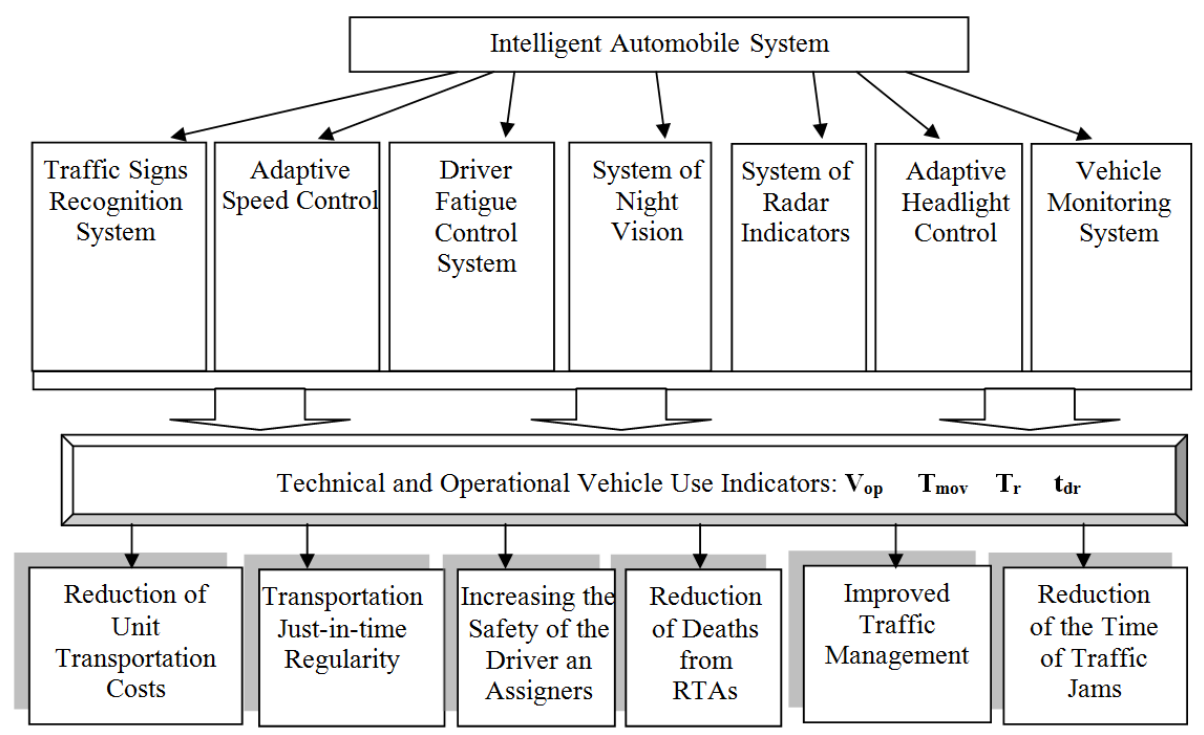

Fig. 4. Intelligent Automobile Systems.

\section{Theory}

Effective traffic flow management includes ensuring the safe use of vehicles and the efficient organization of the transport process. Quality and quantity indicators should be optimally correlated, measured and optimized. Quantitative evaluation criteria include: delay time, number of stops along the way, queue length, average speed, movement time, time of delivery of goods, fuel consumption. The main criterion for the effectiveness of ITS algorithms is the delay of one vehicle in the flow. It follows that each delay of one vehicle in the flow leads to the formation of a queue, where in the future there is a traffic jam and there are losses of time on the transportation route. Timely response and adjustment of the 
vehicle route of movement on the basis of ITS use positively affects the quality of the transportation process in general $[17,18,19]$.

The street network monitoring system is designed to manage the traffic flow, increase the capacity of the street network, prevent traffic jams, reduce the delays of vehicles in motion, improve the traffic safety, optimize the transportation process, inform the traffic participants about the situation on the transport network and offer optimal routes for traffic, as well as ensuring uninterrupted movement of fixed route passenger transport (Figure 5) $[20,21]$. Optimization of the transportation process is to optimize the quality indicators of freight and passenger transportation (Figure 6) [22, 23, 24].

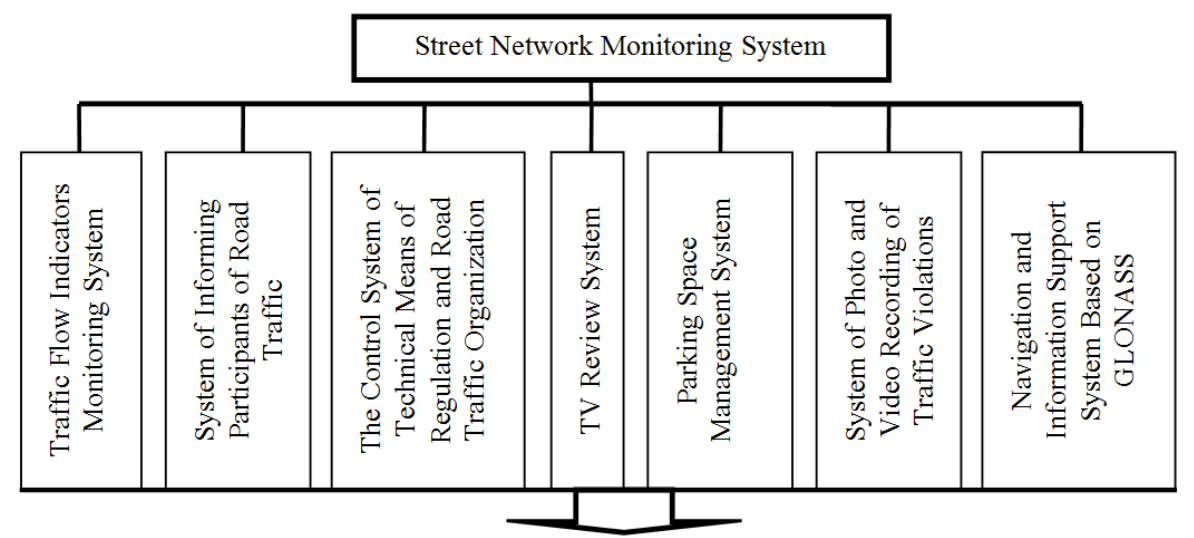

Parameters of Traffic Flow, Operation of the Vehicle and Route of Movement: NTF, qTF, $T_{\text {mov, }} L_{\text {tot. }} V_{\text {av }}$

Fig. 5. The Street Network Monitoring System.

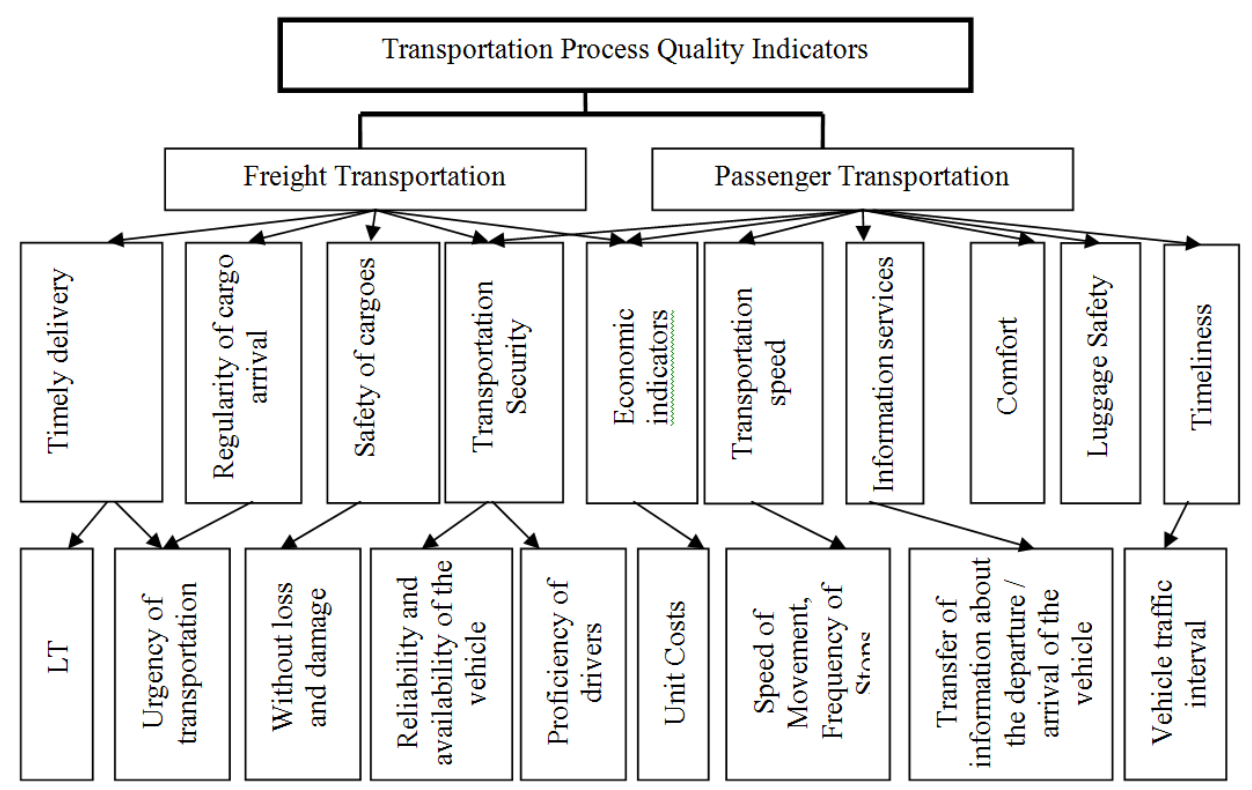

Fig. 6. Quality Indicators of the Transportation Process. 
Within the ITS, the following traffic quality indicators can be improved: timeliness of transportation, transport security, economic indicators, speed of transportation, information support.

\section{Results and discussions}

The results of the conducted research are recommended to be used when developing the digital model of the "Smart City" system. This will allow to harmonize traffic flows and implement a rationalized development of the passenger transport route network, control the movement of vehicles on the street-road network, ensure the functioning of the geoinformation resource for to ensure the movement of unmanned vehicles and traffic management.

The introduction of smart infrastructure and services is a promising process [25]. The benefits of a smart city to communities are unlikely to be rapid and likely to increase in the first phase. Information and communication technologies and the Internet of Things for urban infrastructure and smart expansion of services, while offering great financial benefits.

\section{Conclusion}

However, all current systems related to vehicles or infrastructure have limitations in function and efficiency, despite the fact that they have been shown to contribute to a more efficient and safe movement that does not damage the environment [26, 27].

Thus, the functioning of the intelligent transport system provides a transport balance between the existing street network capacity and the demand for road traffic, the efficient car use and the improvement of the quality of public transport services.

\section{References}

1. Carmona, Matthew. Public places, urban spaces: the dimensions of urban design. Routledge (2010)

2. Kitchin, Rob. "Big Data, new epistemologies and paradigm shifts." Big Data \& Society 1.1: 2053951714528481. (2014)

3. Misuraca, Gianluca, Francesco Mureddu, and David Osimo. "Policy-making 2.0: Unleashing the power of big data for public governance." Open government. Springer, New York, NY, pp. 171-188 (2014)

4. Albino, Vito, Umberto Berardi, and Rosa Maria Dangelico. "Smart cities: Definitions, dimensions, performance, and initiatives." Journal of Urban Technology 22.1, pp. 321 (2015)

5. I. Lakshmi, "A literature survey on Big Data Analytics in Service Industry." International Journal of Engineering and Computer Science 5.4 (2016). Breslin, J., Decker, S. (2007)

6. Dimitrakopoulos, George, and George Bravos. Current Technologies in Vehicular Communication. Springer (2016)

7. Psomakelis, Evangelos, et al. "BIG IOT AND SOCIAL NETWORKING DATA FOR SMART CITIES."

8. Chong, Hon Fong, and Danny Wee Kiat Ng. "Development of IoT device for traffic management system." In Research and Development (SCOReD), 2016 IEEE Student Conference on, pp. 1-6. IEEE (2016) 
9. M.A. Korablin, S.V. Smirnov, Inheritance of properties in the problems of objectoriented programming in Modula-2, Programming, 4, pp. 38-43 (1990)

10. L. Ya. Kozhukhovskaya, V.N. Baskov, A.V. Ignatov, Modular Management of Indicators of Efficiency and Safety of Transportation Processes, Transportation Research Procedia, 20, pp. 361-366 (2017)

11. T.I. Mikheeva, S.V. Mikheev, Using the principles of object-oriented design of an intelligent transport system, Bulletin of SamSTU, 34, pp. 141 - 148 (2005)

12. T. Liebig, N. Piatkowski, Ch. Bockermann, K. Morik, Dynamic route planning with real-time traffic predictions, Information Systems, 64, pp. 258-265 (2017)

13. T.I. Mikheeva, S.V. Mikheev, Models of inheritance in the traffic management system, Information technology, 7, pp. 50-54 (2001)

14. A.P. Artynov, G.A. Kondratiev, Managing the interaction of transport systems, Moscow: Nauka,197 p. (1986)

15. H. Inose, T. Hamada, Traffic management: Trans. with English, Moscow: Transport, 248 p. (1983)

16. G.I. Klinkovshteyn, Organization of road traffic, Moscow: Transport, 240 p. (1982)

17. E.I. Vidmanova, V.N. Baskov, Assessment of the level of adaptation of the traffic flow to the road network, Bulletin of the Saratov State Technical University, 71, Part 2, pp. 364-368 (2013)

18. M. Aslani, M.S. Mesgari, .M. Wiering, Adaptive traffic signal control with actor-critic methods in a real-world traffic network with different traffic disruption events , Transportation Research Part C: Emerging Technologies, 85, pp. $732-752$ (2017)

19. A. Danilevičius, M. Bogdevičius, Investigation of Traffic Light Switching Period Affect for Traffic Flow Dynamic Processes Using Discrete Model of Traffic Flow, Procedia Engineering, 187, pp. 198-205 (2017)

20. R. Li, M. Guo, Effects of odd-even traffic restriction on travel speed and traffic volume: Evidence from Beijing Olympic Games, Journal of Traffic and Transportation Engineering (English Edition), 3, Iss. 1, pp. 71-81 (2016)

21. J. Chen, L. Ming, R. Jiang, Effects of the amount of feedback information on urban traffic with advanced traveler information system, Physics Letters A, 381, 35, pp. 2934-2938 (2017)

22. A.V. Ignatov, V.N. Baskov, Categorization of sections of the street-road network according to the degree of risk of traffic congestion, taking into account the speed intervals, The world of transport and technological machines, 3 (54), pp. 53-60 (2016)

23. S. Hohmann, J. Geistefeldt, Traffic Flow Quality from the User's Perspective, Transportation Research Procedia, 15, pp. 721-731 (2016)

24. E.I. Isaeva, V.N. Baskov, On the issue of the estimation of the traffic flow using technical means of measurement, Actual questions of organization of road transport and traffic safety: II intern. scientific-practical. Conf., Saratov: SSTU (2017)

25. V. Zyryanov, V. Kocherga, I. Topilin, Investigation of Dependencies between Parameters of Two-component Models of the Kinetic Theory of Traffic Flow and Traffic Characteristics, Transportation Research Procedia, 20, pp. 746-750 (2017)

26. T.I. Mikheeva, S.V. Mikheev, Methods and tools for the design of traffic control systems , Intellectual systems: Tr. The Sixth International Symposium (INTELS '2004) ed. K.A. Pupkov. - Moscow: RUSAKI, pp. 406 - 409 (2004)

27. A.V. Ostroukh, N.E. Surkov, Intellectual information systems and technologies: monograph , Krasnoyarsk: Scientific and Innovation Center, 370 p. (2015) 